\title{
Research on Flow and Temperature Fields in Aircraft Engines
}

\author{
Guochang Zhao ${ }^{1, *}$, Daniel X. Zhao ${ }^{2}$, Jing $\mathrm{Li}^{3}, \mathrm{Xia} \mathrm{Du}^{3}$, Xianyi Tong ${ }^{3}$, Chunlei Zhao ${ }^{3}$, Jingru Kong ${ }^{3}$, \\ Tiandong $\mathrm{Lu}^{3}$, Lei $\mathrm{Cao}^{3}$ and Liping Song ${ }^{1}$
}

${ }^{I}$ Tianjin Key Laboratory of Civil Aircraft Airworthiness and Maintenance, Civil Aviation University of China, Tianjin 300300, China; ${ }^{2}$ Department of Electrical Engineering and Computer Science, University of Michigan-Ann Arbor, MI 48109, USA; ${ }^{3}$ Faculty of Aerospace Engineering, Shenyang Aerospace University, Shenyang 110136, China

\begin{abstract}
The similarity criteria which ensure the similarity between flow and temperature fields of both the lowtemperature turbine cavity model and the actual high-temperature turbine cavity are derived and then verified using numerical simulation of two different sized rotor-stator system turbine cavities. The analytical solution of threedimensional dimensionless velocity distribution and the numerical solutions of both the three-dimensional dimensionless velocity and temperature distributions of the free disk flow field are obtained. Using these solutions as a foundation, the analytical solutions of the dimensionless temperature distribution within the boundary layer of the isothermal and nonisothermal free disk model are obtained. The numerical and analytical solutions of velocity and temperature are compared to ensure that the solutions are consistent with each other. The analytical solutions of the dimensionless velocity and temperature fields within the laminar boundary layer of the wedge flow are obtained. The approximation solutions of the dimensionless temperature of the wedge flow under subsonic speed and isothermal wall boundary conditions and the dimensionless temperature of the wedge flow under supersonic speed and adiabatic wall boundary conditions are provided. The velocity and temperature distributions of the airflow outside the strut are obtained through numerical simulation and the pattern of changes in the velocity and temperature within the laminar boundary layer of the strut is revealed. Numerical simulation on gas jet cooling and regenerative cooling for the strut are simulated and shown to be effective for thermal protection. Using a strut with rough internal surface and aviation kerosene as the cooling medium is effective at cooling. The experiment fixture used to simulate the outlet temperature of the combustor and the experimental fixture used to simulate the dynamic temperature of the compressor are designed and built. The dynamic temperature of the compressor airflow is measured using combined thermocouples. Experimental results show that the dynamic error caused by the thermal inertia of the thermocouple can be eliminated by the compensation algorithm.
\end{abstract}

Keywords: Aero-engine, boundary layer, combustor, compressor, dynamic temperature measurements, flow field, jet cooling, optical fiber temperature measurement, regenerative cooling, similarity, strut, temperature field, turbine cavity, wedge flow.

\section{INTRODUCTION}

Research on flow field and temperature field has helped to optimize design and performance of the aircraft engines. Our research group focuses on the flow and temperature fields of the aircraft engines and has conducted a series of studies. Research has been conducted on flow and temperature fields of turbine disk cavity, temperature field in the wedge flow boundary layer, thermal protection of strut, temperature measurement of the compressor with combined thermocouples, and temperature measurement of the combustor exit with optical fiber. This work on flow and temperature fields is crucial for key parts of aircraft engines. Theoretical analysis and numerical simulations have been applied on this research and experimental validation is being carried out.

*Address correspondence to this author at the Tianjin Key Laboratory of Civil Aircraft Airworthiness and Maintenance, Civil Aviation University of China, Tianjin 300300, China; Tel: 024-89728820; Fax: 024-89728820; Email:shdg23@gmail.com

\section{SIMILARITY STUDY ON FLOW AND TEMPE- RATURE FIELDS OF TURBINE DISK CAVITY}

\subsection{Significance of Research}

It is preferable to conduct experiments under actual or similar operating conditions to investigate the flow and temperature fields of the turbine disk cavity in aircraft engines. But there are significant drawbacks regarding these high temperature experiments in terms of cost, accuracy of measurements, risk and difficulty. These drawbacks can easily be overcome by using experiments under low temperature conditions but how accurately the low temperature experiments reflect the flow and heat transfer of the turbine disk cavity under high temperature condition is debated. Work based on similarity theory has been carried out to ensure that low temperature experiment results are applicable towards high temperature experiments. According to non-dimensionalized flow and heat transfer governing equations, as long as some similarity criterions are equal between low temperature experiments and high temperature, 
experiments the dimensionless velocity and temperature fields under low and high temperature conditions will be the same. This conclusion has been confirmed by numerical simulations.

\subsection{Simulation of Laminar Flow and Heat Transfer in a Rotor-stator System}

After non-dimensionalizing the flow and heat transfer governing equations of a rotating system, the dimensionless numbers $R e, R e_{\omega}, G r_{\omega}$ are deduced as the similarity criteria. Based on the fact that the geometry and dimensionless boundary conditions are similar, the dimensionless velocity and temperature fields under low and high temperature conditions are similar when $R e, R e_{\omega}$ and $G r_{\omega}$ are equal to their counterparts. In order to validate the conclusion, two models shown in Fig. (1) and Table 1 were established.

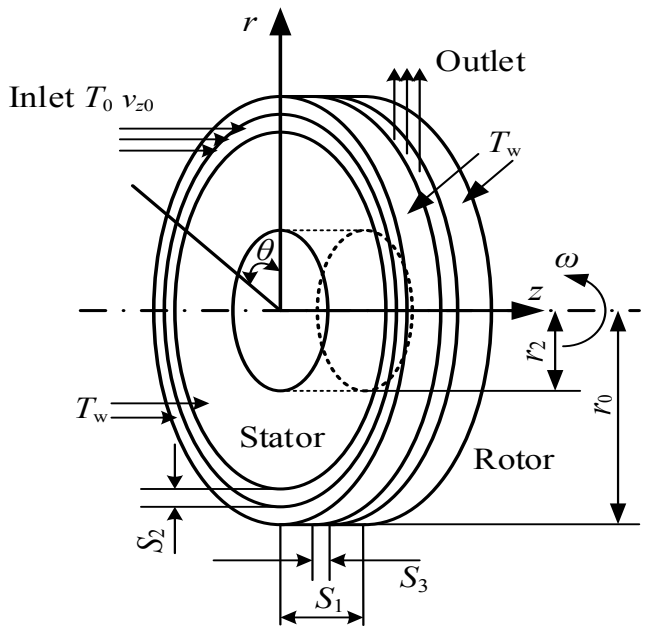

Fig. (1). Schematic diagram of turbine cavity.

Model 2 is a $1 / 2$ of Model 1 in terms of size and both models have equal $\mathrm{Re}, R e_{\omega}$ and $G r_{\omega}$ parameters. Fluent software has been applied to simulate the laminar flow and heat transfer of the two models. Figs. $(2,3)$ show the dimensionless velocity and temperature contours on $z-r$ plane of the two models respectively. In each figure, the contours are nearly identical which means that the flow and temperature fields are similar. Near the inlet, outlet and rotating disk, the dimensionless velocity of airflow is relatively bigger. Near the inlet, dimensionless temperature of airflow is the minimum and temperature increases as the distance between the airflow and inlet further increases. The conclusion can be drawn that if the geometry and boundary conditions are similar, as long as $R e, R e_{\omega}$ and $G r_{\omega}$ of the two models are equal respectively, the flow and temperature fields of turbine cavity can be simulated even under low temperature conditions.

\section{NUMERICAL AND ANALYTICAL SOLUTIONS OF FLOW AND TEMPERATURE FIELDS OF A FREE DISK}

\subsection{Significance of Research}

Free disk flow refers to the fluid flow field which is induced by the rotating disk with certain angular velocity in infinite space and fluid far away from the rotating disk remains still. Although the disk structure differs from free disk in practical aircraft engines, investigating the velocity and temperature distribution of free disk is the first step to understanding the distribution in an actual complex rotating system such as turbine cavity. Reference [1] even deemed that for some cases, rotating disk in a rotor-stator system can be simplified as free disk. Therefore, it is reasonable to study the flow and heat transfer characteristics of the free disk before studying the actual aero-engine turbine cavity. (a) Model 1

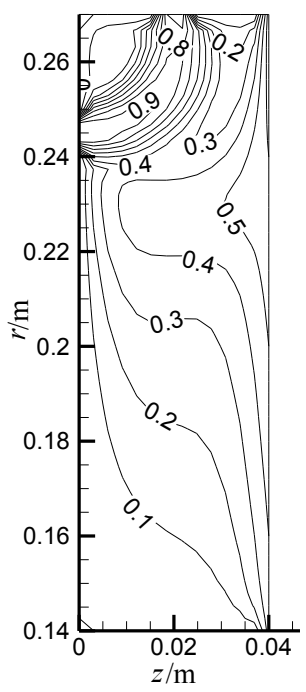

(b) Model 2

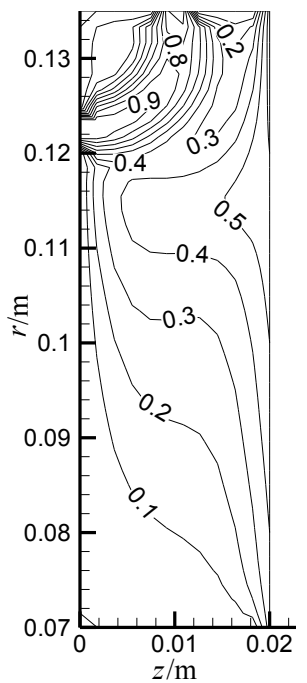

Fig. (2). The dimensionless velocity contours on $z-r$ plane. (a) Model 1

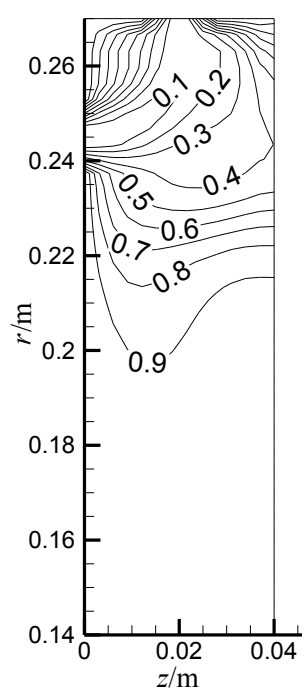

(b) Model 2

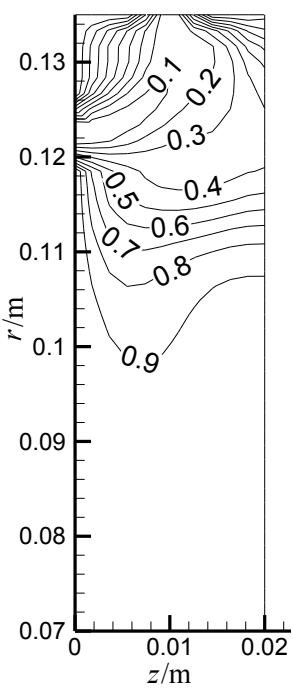

Fig. (3) The dimensionless temperature contours on $z-r$ plane.

\subsection{Results}

Von Karman [2] was the first great researcher to obtain a three dimensional velocity analytical solution of free disk. Cochran [3] corrected a flaw of Von Karman's solution and obtained a more accurate analytical solution. Based on existing literature, a three dimensional velocity analytical solution of free disk is obtained with MATLAB and a numerical solution which matches very well with the analytical solution has been derived. Fig. (4) represents 
Table 1. Model parameters.

\begin{tabular}{|c|c|c|c|c|c|c|c|c|}
\hline & $\begin{array}{l}\text { Outer } \\
\text { Radius } \\
\mathbf{r}_{0} / \mathbf{m m}\end{array}$ & $\begin{array}{c}\text { Rotor-to-Stator } \\
\text { Distance } \\
\mathrm{S}_{1} / \mathbf{m m}\end{array}$ & $\begin{array}{l}\text { Inlet } \\
\text { Width } \\
\mathbf{S}_{2} / \mathbf{m m}\end{array}$ & $\begin{array}{l}\text { Outlet } \\
\text { Width } \\
\mathbf{S}_{3} / \mathbf{m m}\end{array}$ & $\begin{array}{c}\text { Inner } \\
\text { Radius } \\
\mathbf{r}_{2} / \mathbf{m m}\end{array}$ & $\begin{array}{c}\text { Inlet } \\
\text { Velocity } \\
\mathbf{v}_{\mathbf{z o}} /\left(\mathbf{m} \cdot \mathbf{s}^{-1}\right)\end{array}$ & $\begin{array}{l}\text { Angular } \\
\text { Velocity } \\
\omega /\left(\operatorname{rad} \cdot \mathbf{s}^{-1}\right)\end{array}$ & $\begin{array}{c}\text { Temperature of Stator and } \\
\text { Outer Edge of Rotor-Stator } \\
\mathbf{T}_{\mathrm{w}} / \mathbf{K}\end{array}$ \\
\hline Model 1 & 270 & 40 & 10 & 10 & 140 & 0.12 & 0.3 & 1793.15 \\
\hline Model 2 & 135 & 20 & 5 & 5 & 70 & 0.24 & 1.2 & 351.05 \\
\hline
\end{tabular}

schematic diagram of velocity distribution within the boundary layer of the free disk. Fig. (5) illustrates a line plot depicting the variation of the dimensionless velocity and dimensionless temperature of the free disk flow along dimensionless axial height where the dimensionless variables $F, G, H$ and $\Theta$ denote radial velocity, tangential velocity, axial velocity and temperature. Fig. (6) shows comparison of numerical solution and analytical solution of the three dimensionless velocity components.

To date, there exists only analytical solution of the dimensionless velocity distribution. Due to the complexity of solving nonlinear ordinary differential equations, the analytical dimensionless temperature solution of free disk has not yet appeared. On the basis of analytical solution of the dimensionless velocity, the governing equations of free disk with both isothermal and non-isothermal boundary conditions respectively and originally obtained numerical solution and analytical solution of dimensionless temperature are shown in Fig. (7) (isothermal free disk: $n=0$, nonisothermal free disk: $n=2$ ), and it has been observed that the analytical solution and numerical solution match very well.

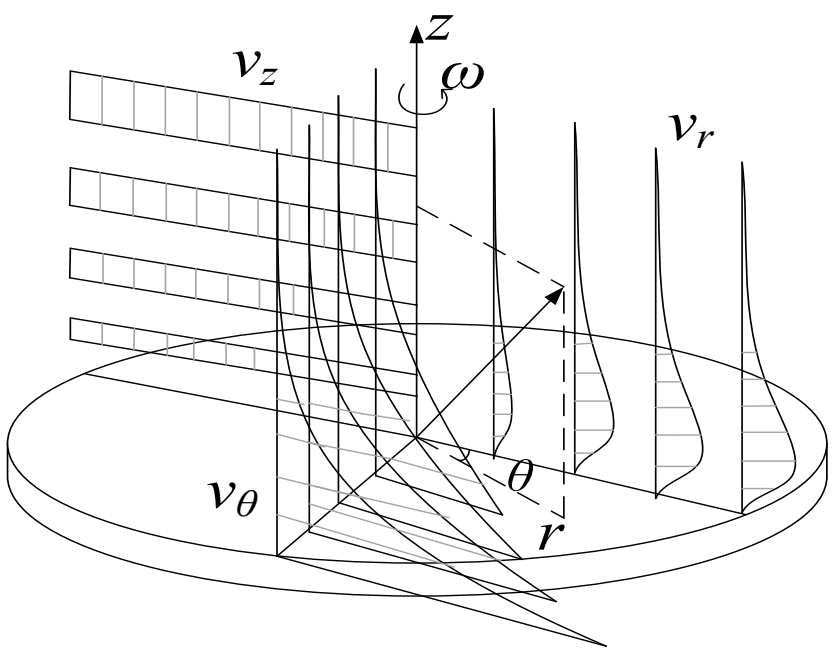

Fig. (4). Schematic diagram of velocity distribution within the boundary layer of the free disk.

\section{TEMPERATURE FIELD OF STRUT AND TEMPE- RATURE PROFILE IN LAMINAR BOUNDARY LAYER OF WEDGE FLOWS}

\subsection{Significance of Research}

Strut is an important component in the combustor. Its main role involves mixing up of the fuel with the supersonic incoming stream and stabilizing combustion in the chamber, acting as fuel injector and flame stabilizer. Structure and position of the strut in a scramjet are shown in Fig. (8).

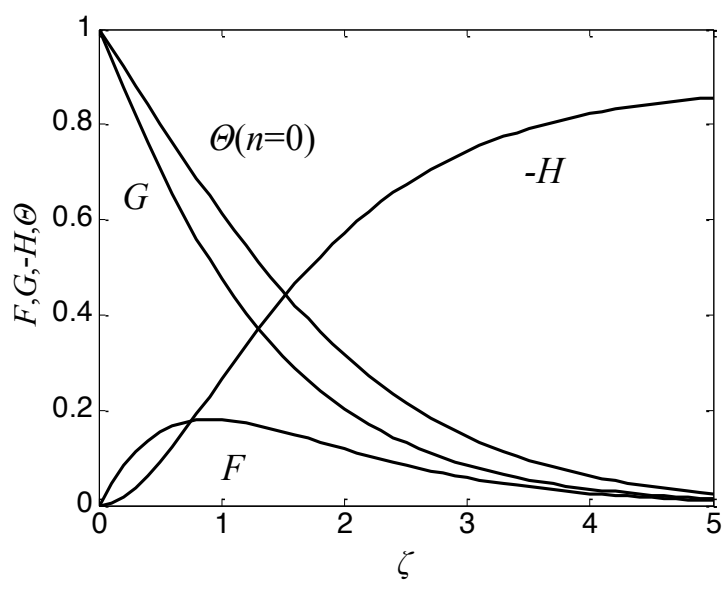

Fig. (5). Variation of the dimensionless velocity and dimensionless temperature of the free disk flow along dimensionless axial height.

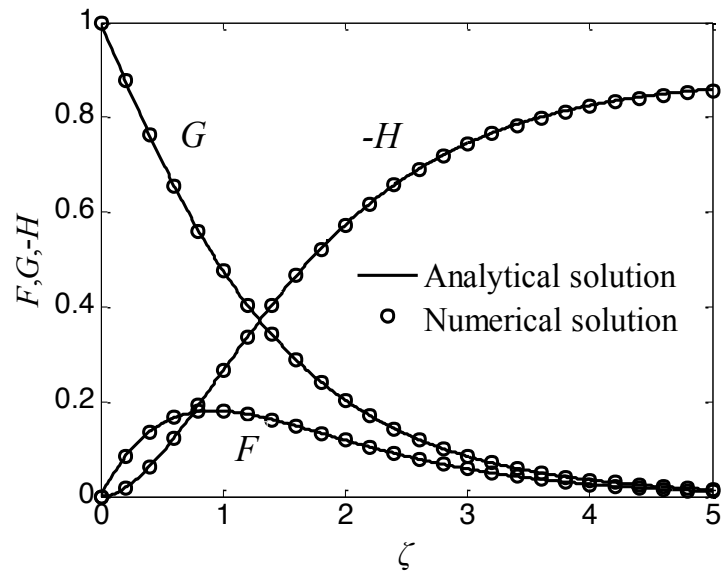

Fig. (6). Comparison of numerical solution and analytical solution of the dimensionless velocity.

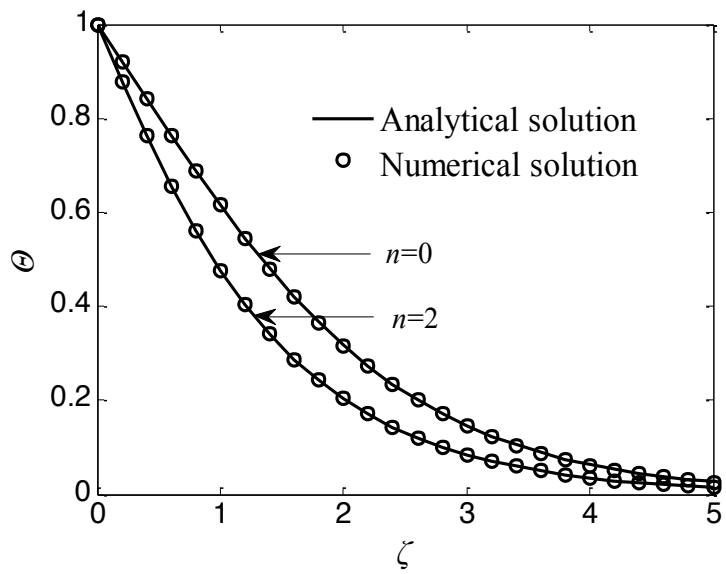

Fig. (7). Comparison of numerical solution and analytical solution of the dimensionless temperature. 


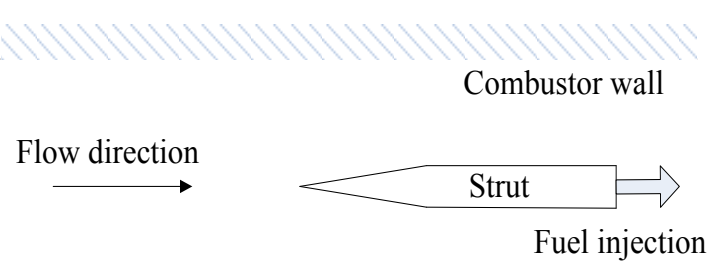

Fig. (8). Schematic diagram of the position of the strut in a scramjet.

There is a high-temperature zone in the leading edge stagnation region of the strut where thermal ablation easily occurs due to aerodynamic heating and the high local heat flux density. Moreover, the strut is prone to deformation and strength decline under extremely high temperatures. Strut thermal protection is particularly important in prolonging the working life of the engine. Since the shapes of strut in both scramjet and aircraft engines are similar, the aerodynamic heat environment is only slightly different. The flow and heat transfer research on aircraft engine rectifier strut can refer to research methods and findings of flow and heat transfer research completed under a premise of subsonic flow around the strut.

\subsection{Results}

\subsubsection{Thermal Environment Around the Strut}

CFD software is used to simulate the external flow field of scramjet strut under adiabatic wall conditions to obtain the velocity and temperature distribution of the boundary layer flow around the strut as shown in Fig. (9). The results are shown in Figs. $(\mathbf{1 0}, \mathbf{1 1})$. Velocity and temperature contours at the strut leading edge are very intensive and the highest temperature appears at the stagnation point of leading edge. The heat flux distribution on the surface of the strut under isothermal wall conditions has also been studied. Results show that there is a local high heat flux zone around the stagnation point of the strut leading edge. The temperature field in the boundary layer of laminar flow over the strut under conditions of different wedge angles and leading edge radius shows that the smaller the wedge angles or leading edge radius, the higher the temperature of stagnation point and the higher the heat flux around the stagnation point.

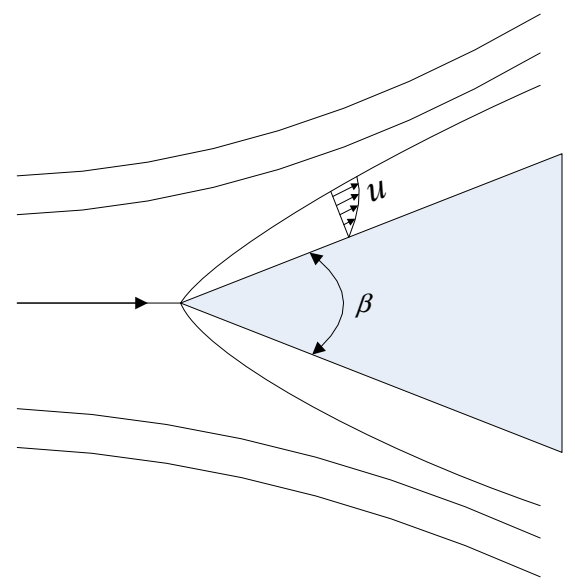

Fig. (9). Schematic diagram of two-dimensional physical model of strut.

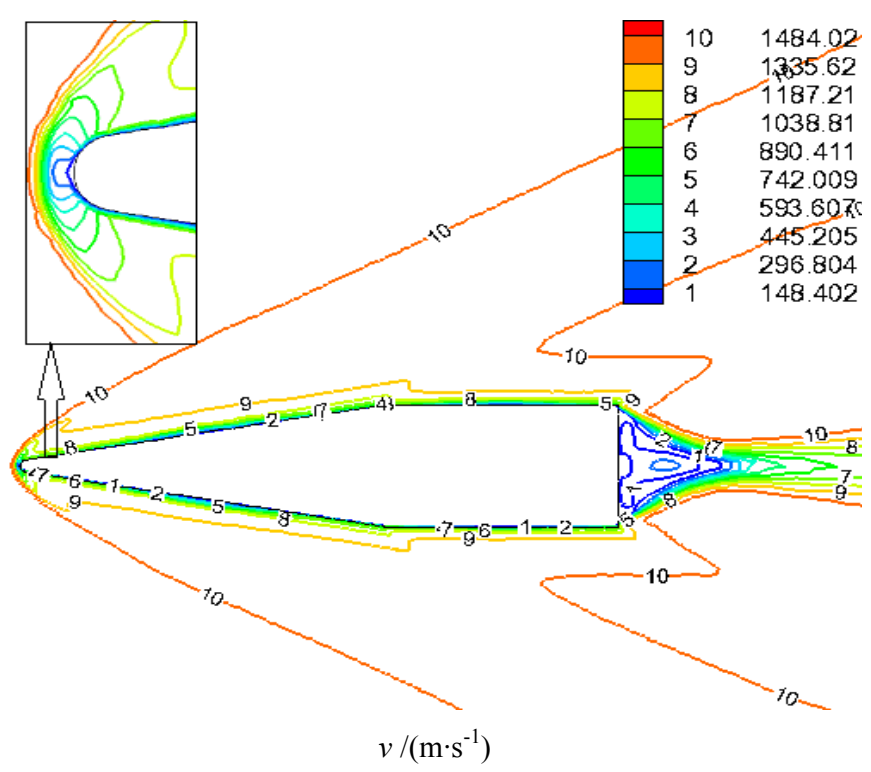

Fig. (10). Velocity contours in the boundary layer around the strut.

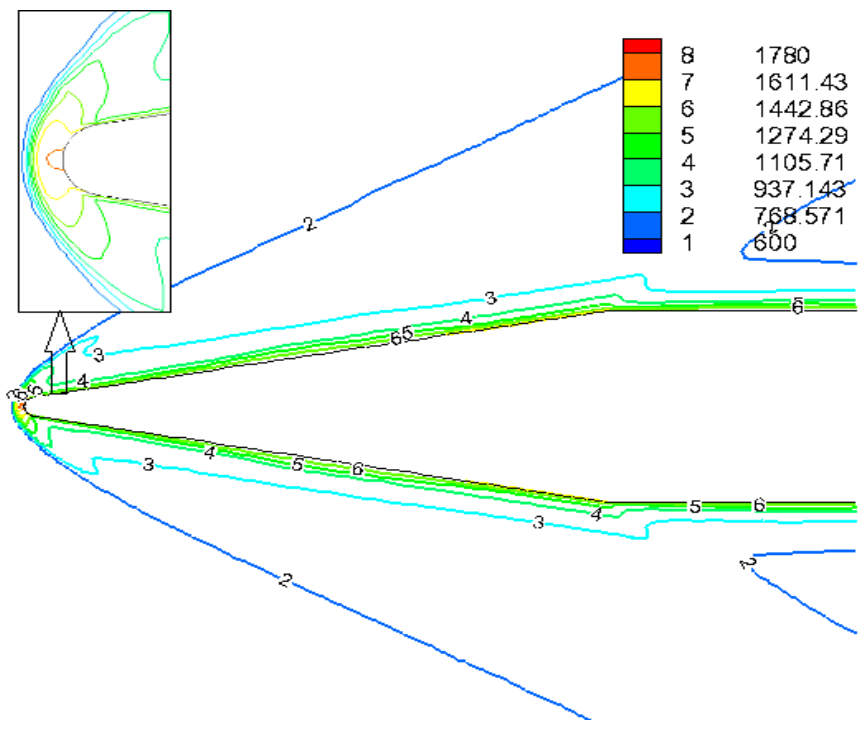

$T / \mathrm{K}$

Fig. (11). Temperature contours in the boundary layer around the strut.

\subsubsection{Velocity and Temperature Fields of Laminar Boundary Layer of Wedge Flows}

In order to study the velocity and temperature distribution of the laminar layer of wedge flows, the dimensionless velocity and temperature field of laminar layer around a strut were programmed. These calculations reflect the variation of dimensionless velocity and temperature field of the laminar layer of strut wedge flow [4]. The fitting polynomial of dimensionless stream function is as follows:

$$
f(\eta)=b_{0} \eta^{5}+b_{1} \eta^{4}+b_{2} \eta^{3}+b_{3} \eta^{2}
$$

where $b_{0}, b_{1}, b_{2}, b_{3}$ are the coefficients of polynomial and $\eta$ is the dimensionless similarity variable. Comparing with other reported fitting formulae, this expression does not include the first order term and the constant term and the number of undetermined coefficients is reduced to four. Dimensionless 
velocity distributions of the laminar layer of wedge flow with different wedge angles are shown in Fig. (12). The dimensionless velocity approaches 1 as $\eta$ increases which reveals how the velocity in the boundary layer changes to the same velocity as the main flow. The larger the wedge angle $\beta$, the more rapidly the dimensionless velocity changes near the wall $(\eta=0)$ as $\eta$ varies and the thinner the boundary layer is. The dimensionless temperature distribution of the laminar boundary layer of the wedge flows under constant wall temperature of the scramjet strut under subsonic condition is shown in Fig. (13).

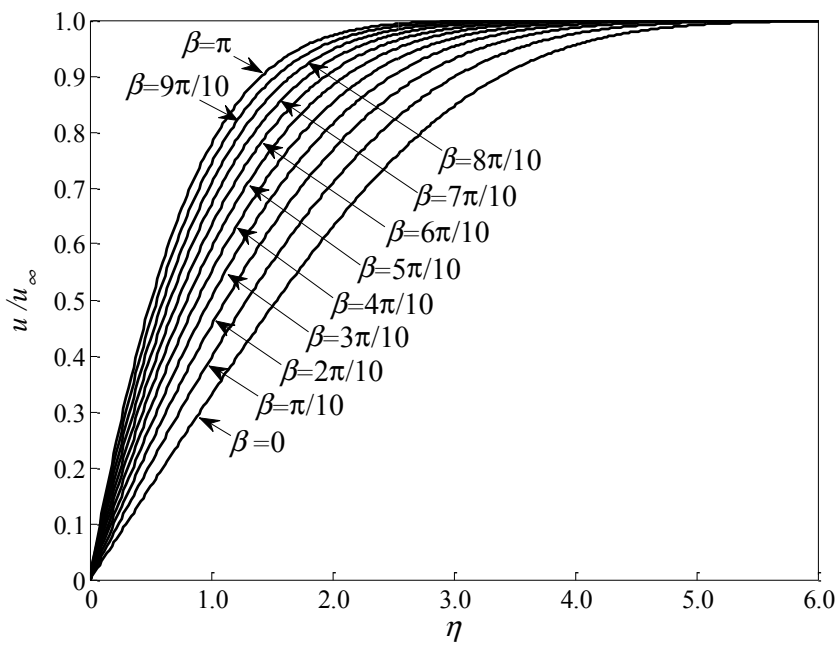

Fig. (12). The dimensionless velocity with different $\beta$.

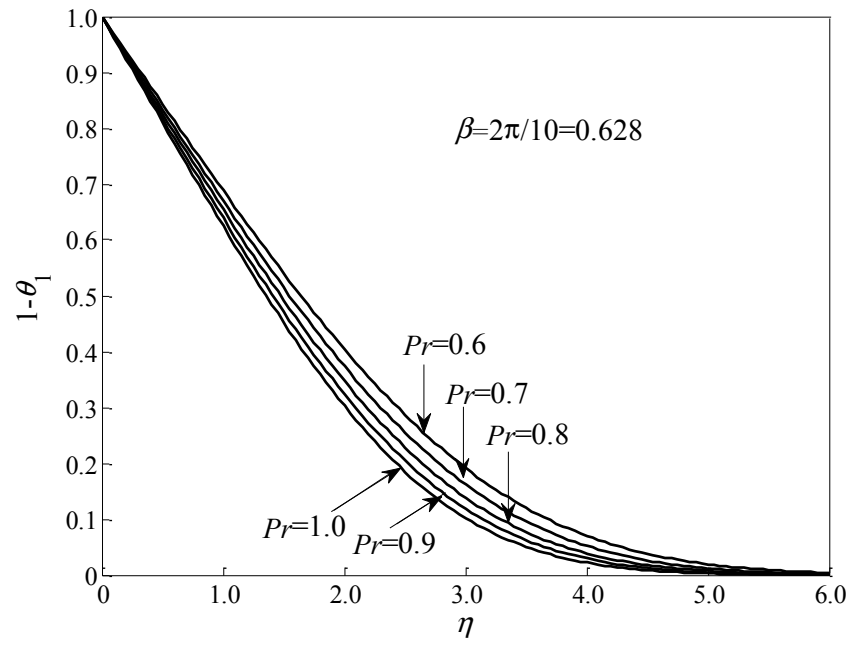

Fig. (13). The dimensionless temperature distribution of the laminar boundary layer of the wedge flow under the condition of constant wall temperature.

As shown in Fig. (13), the dimensionless temperature increases to 1 as the similarity variable $\eta$ increases. The larger the Pr number, the more rapidly the dimensionless temperature increases in the zone near the wall and the thinner the temperature boundary layer. The dimensionless temperature distribution in the laminar boundary of a scramjet strut under adiabatic wall and supersonic conditions is shown in Fig. (14). It is shown that the dimensionless temperature of gas in the boundary layer decreases as the similarity variable $\eta$ increases and the temperature near the wall decreases more quickly as $\operatorname{Pr}$ number increases.
On the basis of the above study, the fitting solution of dimensionless temperature in exponential form under isothermal and adiabatic wall conditions is given which reveals how the dimensionless temperature changes as similarity variable $\eta$ and $\operatorname{Pr}$ number vary. As compared with obtaining numerical solutions, the cost and difficulty of computing decrease dramatically as reported in reference [4].

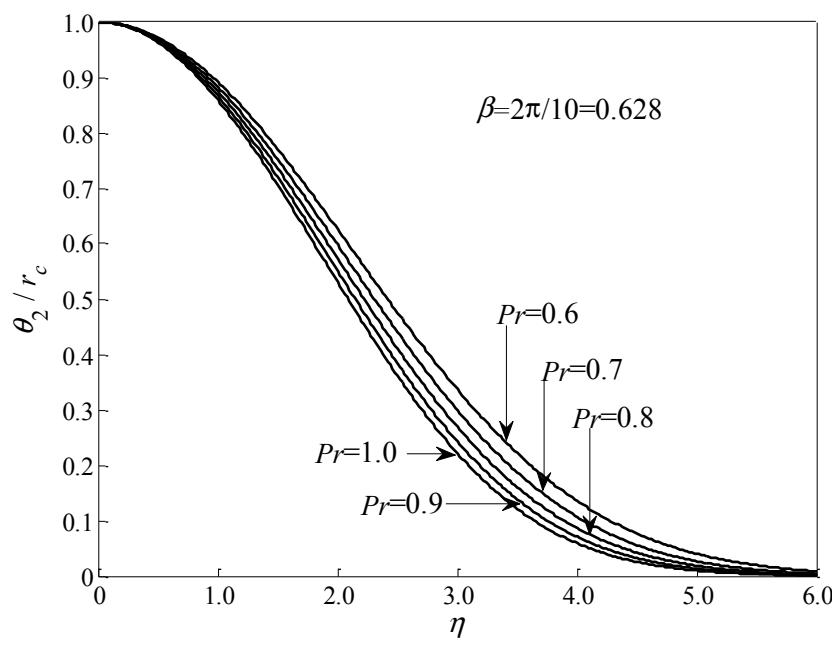

Fig. (14). The dimensionless temperature distribution of the laminar boundary layer of the wedge flows under the adiabatic wall conditions.

\subsubsection{Thermal Protection of Strut}

There are two ways to cool the strut: active cooling and passive cooling. Active cooling includes regenerative cooling and gas jet cooling. Regenerative cooling embeds cooling channels inside the metal structures which need to be cooled. The coolant goes into one side of the cooling channel and goes out of the other side in order to cool the strut. This cooling method can also be used in the wall of combustion chamber of scramjet [5]. If the fuel is used as regeneration coolant, it is warmed up to facilitate the ignition [6]. The strut jet cooling has been studied under conditions of Mach number over 6 . When gas ejects from the narrow slit, it expands and temperature decreases quickly so the maximum temperature zone moves away from the leading edge of strut. The cooling gas from narrow slit mixed with incoming stream forms an obvious gas film on the leading edge and the temperature in this film is apparently lower than outer flow which plays a key role in thermal protection [5-9]. The coolant can be gas, water or fuel. A regenerative cooling model of strut has been established and numerical simulation has been completed. It is found that for rough channel, the contact area of coolant and the wall is larger, so coolant can take away more heat.

\subsection{Conclusion and Prospect}

The temperature gradient in the leading edge of strut is bigger and the highest temperature appears at the stagnation point. The temperature of stagnation point increases with a decrease in radius or wedge angle. The thickness of velocity boundary layer decreases as the wedge angle increases. The fitting solution of dimensionless temperature in exponential form under the condition of constant wall temperature is 
given which reveals how the dimensionless temperature changes as similarity variable $\eta$ and $P r$ number vary. The fitting solution of dimensionless temperature of wedge flow in exponential form under the condition of supersonic adiabatic is also given. As compared with obtaining numerical solutions by solving ODEs, the cost and difficulty of computing decrease dramatically. As the thickness of boundary layer increases, when the wall temperature is lower than thermal isolation wall temperature, the gas temperature of boundary layer increases and then decreases due to viscous dissipation. Gas jet cooling plays a good role in thermal protection. The coolant in the strut with rough channel can take away more heat. Further planned research includes the following: the optimization problem of regeneration cooling will be studied with neural network algorithm; effects of various factors on strut regeneration cooling will be studied with CFD software; optimization of the strut structure to obtain higher cooling efficiency under low fuel flow rate conditions will also be researched. Study on the composite cooling method of $\mathrm{C} / \mathrm{SiC}$ strut will be continued along with building of an experimental platform to simulate the thermal environment of the strut. Experimental study on the flow and heat transfer characteristic of laminar boundary layer over a plate, optimization of the cooling channel for wedge regeneration, experimental study on the flow and heat transfer characteristics of coolant in regeneration cooling channels and experimental study on the flow and heat transfer characteristics of external wedge wall are all possible future areas of research.

\section{GAS TEMPERATURE MEASUREMENT COMBUSTION CHAMBER OUTLET}

AT

\subsection{Significance of Measuring the Gas Temperature at the Combustion Chamber Outlet}

Modern testing technology for aircraft engine is a crucial support of aero-engine propulsion technology and is an important technical part of engine pre-research experimental study and aero-engineering development. Since temperature is one of the key parameters closely related to the performance and operation life of the components with the highest temperature $[10,11]$, accurately measuring the gas temperature at the main combustion chamber outlet can help turbine designers choose cost-effective materials for turbine blade and proper cooling methods [12, 13] and help pilots monitor if the turbine is in correct working state under various flight conditions. Gas temperature measurement at combustion chamber outlet is one of the key techniques in modern aviation engine testing.

\subsection{Advantages of Measuring with Optical Fiber}

In aircraft engines, improving gas temperature at outlet of the main combustion chamber to increase specific thrust is the most direct and effective method to improve the thrust weight ratio of engine. The thrust-to-weight ratio of the fourth generation of turbofan engine is 10 and the average temperature of the gas at the outlet of the main combustion chamber is $1850 \mathrm{~K}$. For the fifth generation, it is 12 and 2000 $\mathrm{K}$ correspondingly and the hotspot temperature can be up to $2600 \mathrm{~K}$. Traditionally, the temperature sensor is platinum- rhodium thermocouple and the gas temperature at outlet of new combustion chamber exceeds the upper temperature limit for conventional thermocouples. Furthermore, high temperature results in some fuel residual and solid mixture. This high speed mixture is ejected from the combustion chamber and can impact the thermocouple, affecting the accuracy and stability of the thermocouple. Due to these factors, traditional thermocouples may not be proper for measuring the extremely high temperatures. Optical fiber sensor has outstanding advantages as an alternative temperature sensor. Optical fibers operate based on Planck's radiation theory on blackbody cavities. The probe is a black body cavity with emissivity close to 1 and the measuring process is hardly affected by the mixture in high velocity stream. The highest temperature which optical fiber sensor can measure is about $3000 \mathrm{~K}$. In addition, the optical fiber sensor has other advantages such as high-temperature durability [11], anti-electromagnetic interference, corrosion resistance, small time constant, high sensitivity, low-cost, light weight, small size and long life. It can measure airflow within a temperature range of $500^{\circ} \mathrm{C}-2000^{\circ} \mathrm{C}$ and speed of $300 \mathrm{~m} / \mathrm{s}$. The steady state temperature measuring accuracy, the system response speed and temperature resolution can be up to $\pm 0.2 \%, 50 \mathrm{kHz}$ and $0.1^{\circ} \mathrm{C}$, respectively. The radiation characteristics of the probe are nearly the same as of the blackbody so the difference in emissivity caused by the temperature and wavelength is negligible [14, 15]. Fig. (15) shows the principle diagram of temperature measurement system using fiber optical sensor.

When the probe of the optical fiber sensor is placed at the outlet of the combustion chamber, the blackbody cavity reaches thermal equilibrium with the gas flow. The thermal radiation generates optical signal which is transmitted to the wavelength division multiplexer (WDM) through the sapphire high temperature optical fiber and the quartz conduction optical fiber.

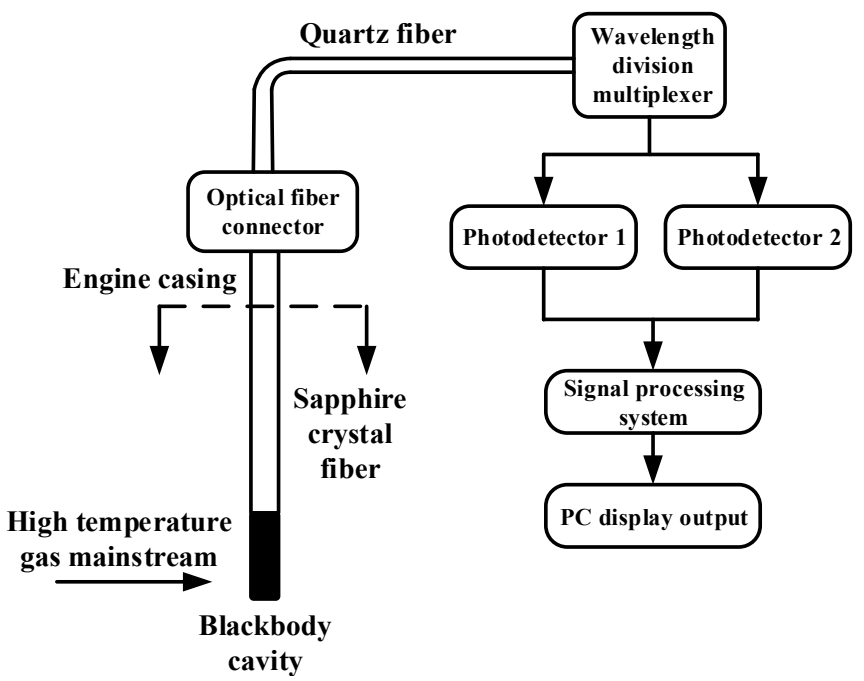

Fig. (15). The principle diagram of temperature measurement system using fiber optical sensor.

The single way optical signal is divided into two ways by a filter and converted into an electrical signal by the photodetector. Afterwards, the electrical signal is amplified and converted in the $\mathrm{A} / \mathrm{D}$ converter to be inputted into the computer. At last, the electrical signal is compensated and 


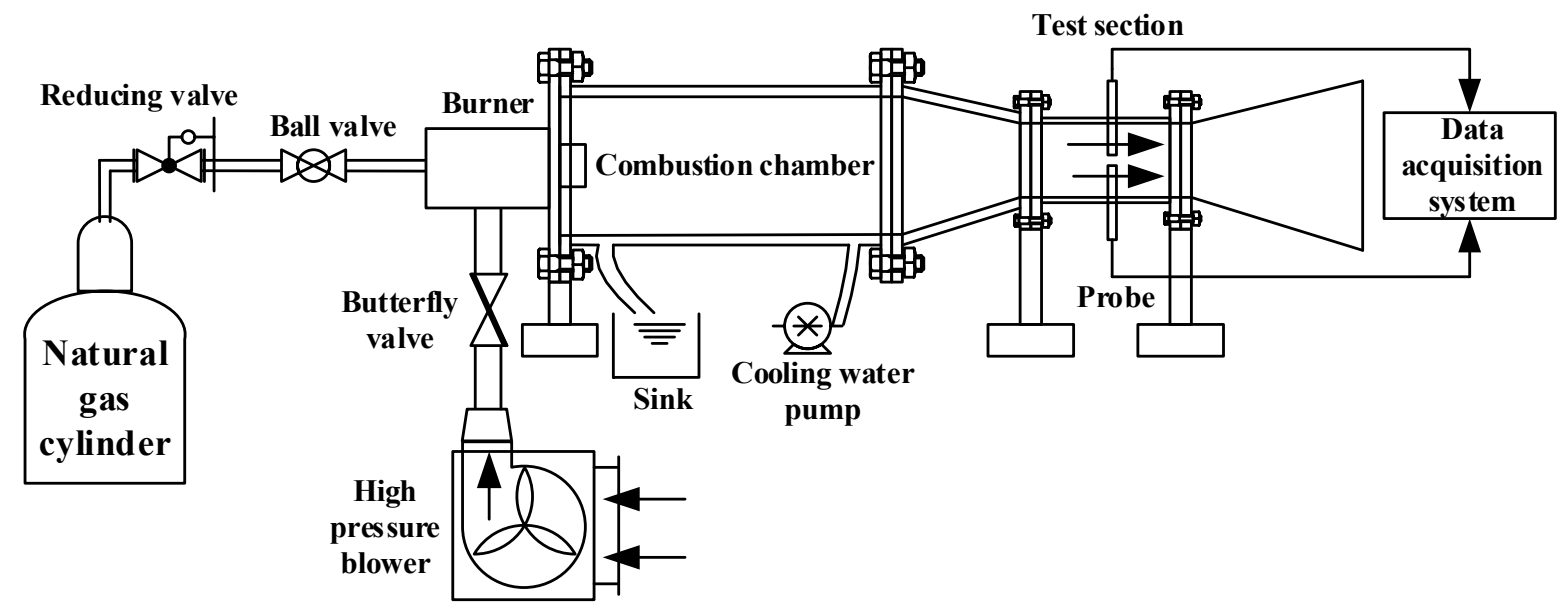

Fig. (16). Schematic diagram of the experimental set-up for simulating the gas temperature at the combustor outlet.

goes through the colorimetric operated processing. After the above processing, the measured temperature is displayed and outputted. As shown in Fig. (16), the test bench can provide $1500 \mathrm{~K}-1700 \mathrm{~K}$ temperature hot gas with the Mach number of gas flow of 0.4 and it can simulate the actual thermal environment of the outlet of a combustion chamber.

\section{COMPRESSOR TEMPERATURE MEASUREMENT}

\subsection{Significance of the Compressor Temperature Measurement}

Currently, more than $80 \%$ [16] calculation error of compressor efficiency is caused by the error of compressor temperature measurement. The accuracy of compressor temperature measurement directly affects the calculation of the compressor efficiency. Distortion caused by temperature and pressure in the compressor affects the normal operation of the engine with the temperature distortion taking precedence over pressure distortion. Thus, it is very important to improve the aircraft engine performance by reducing equivalent time constant of the temperature measuring device and dynamic temperature measurement error $[17,18]$.

\subsection{Two-Thermocouple Method}

Two-thermocouple method uses two thermocouples to simultaneously output two signals which are transmitted, processed and stored. The dynamic temperature value of point measurement can be obtained with help of the temperature compensation program for dynamic error compensation [19, 20]. Test device is shown in Fig. (17). Three thermocouples with different sizes are mounted with an interval angle of $90^{\circ}$ in a stainless steel pipe,; the role of one thermocouple is to monitor temperature fluctuation and the other two are used as the combined thermocouples. The high temperature airflow is provided by the hot airflow gun. $T_{g 1}, T_{g 2}$ denote the real ambient temperature values of the measured medium. $T_{m 1}, T_{m 2}$ are the measured values which follow the following relationship:

$$
\begin{aligned}
& T_{g 1}=T_{m 1}+\tau_{1} \frac{\partial T_{m 1}}{\partial t} \\
& T_{g 2}=T_{m 2}+\tau_{2} \frac{\partial T_{m 2}}{\partial t}
\end{aligned}
$$

where $\tau$ is time constant. Using the least square method, the time constants of the two thermocouples could be obtained due to the fact that the measured medium temperature around two thermocouples is nearly the same. The real dynamic temperature of the measured medium could be obtained by using the above equations.

Results show that dynamic error due to thermal inertia can be eliminated when the signal noise is eliminated.

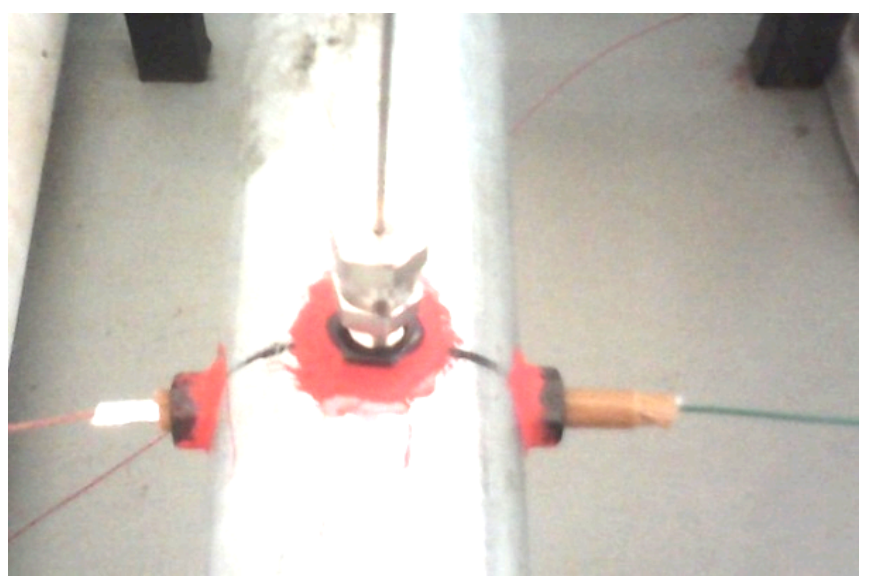

Fig. (17). The experimental set-up of combined thermocouples.

\section{CONCLUSION}

Research has been conducted on key issues such as the velocity and temperature field of the compressor, combustor and turbine cavity of the aero-engine such as the inlet temperature distortion which has great influence on the compressor, the low-temperature experiment simulating the high-temperature turbine cavity experiment and the thermal protection for the strut. No examination has been carried out on analytical solutions of dimensionless temperature of free 
disk in existing literature. Concise dimensionless temperature approximate solutions for the laminar boundary layer of the subsonic wedge flow under the boundary condition of constant wall temperature are given in exponential form. These solutions reveal how the dimensionless temperature changes as similarity variables $\eta$ and $\operatorname{Pr}$ change. The dimensionless temperature approximate solutions for the laminar boundary layer of the supersonic wedge flow under the boundary condition of adiabatic wall are also given in exponential form. These approximate solutions reduce the calculation cost and difficulty significantly while maintaining a high degree of accuracy. Theoretical research has achieved results and related experiments are being conducted. Theoretical research and experiments will continue to be carried out with flow field visualization and temperature measurements for the combustor outlet and the compressor, velocity and temperature measurements and the verification of similarity for the turbine cavity.

\section{CONFLICT OF INTEREST}

The authors confirm that this article content has no conflict of interest.

\section{ACKNOWLEDGEMENTS}

This research is supported by Scientific Research Foundation for Liaoning Province Pandeng Scholars (20132015) and the Aviation Science Foundation of China (20131954004)

\section{REFERENCES}

[1] C. Yuzhang, T. Zhi, and X. Guoqiang, The heat transfer of aeroengine. Beijing University of Aeronautics and Astronautics: Beijing, 2005.

[2] T.V. Kármán, "Über laminare und turbulente Reibung”, ZAMMJournal of Applied Mathematics and Mechanics/Zeitschrift für Angewandte Mathematik und Mechanik, vol. 1, no. 4, pp. 233-252, 1921

[3] W.G. Cochran and S. Goldstein, "The flow due to a rotating disc". Mathematical Proceedings of the Cambridge Philosophical Society, vol. 30, no. 3, pp. 365, 1934

[4] G. Zhao, X. Du and L. Song, "Numerical solutions and fitting solutions of velocity and temperature in Laminar Boundary Layer of Subsonic/supersonic wedge flow", Journal of Aerospace Power, 2014.

[5] H. Wang, G. He and P. Liu, "Optimized design on a new type thermal structure of scramjet combustor". Journal of Propulsion Technology, vol. 30, no. 3, pp. 263-266, 2009

[6] T. Chen, F. Zhong and J. Wang, "Heat transfer analysis and optimized design of actively cooled strut for scramjet applications", In: The $3^{\text {rd }}$ Session of Hypersonic Technology Conference. Jiangsu, Wuxi, 2010, pp. 1-8.

[7] A. Ulas and E. Boysan, "Numerical analysis of regenerative cooling in liquid propellant rocket engines". Aerospace Science and Technology, vol. 24, no. 1, pp. 187-197, 2011

[8] K. Kosaka, T. Kuwahara and H. Okamoto, "Application of carboncarbon composite to scramjet engine". Proceedings of ISABE, vol. 1: pp. 359-364, 1989

[9] Z. Xie and S. Ouyang, "The numerical solution of finite volume method for the N-S equation of viscous flow filed". Journal of Aerodynamic, vol. 14, no. 4, pp. 476-484, 1996

[10] G. Lin, "High-temperature measurement techniques of sapphire crystal fiber". Aero Engine, vol. 4, pp. 58-63, 1998

[11] C. Huang, X. Shi and H. Zhong, "Hot gas temperature measurement technique of gas turbine engine". In: $9^{\text {th }}$ Engine Testing and Test Technology Symposium, Beijing, 2008.

[12] N.-N. Wang, Design and simulation on the fiber-optic sensor of total temperature [D], North University of China: Taiyuan, 2012.

[13] Y. Yang, J. Cai and J. Zhao, "High temperature measurement technology discuss of aviation engine development", Measurement Technology, vol. 28, pp. 46-50, 2008

[14] Z. Liang, G. Chen and Y. Xu, "Research on measurement method of the aeroengine turbine outlet temperature". Chinese Journal of Scientific Instrument, vol. 29, no. 4, pp. 75-77, 2008.

[15] J. Zhu, X. Hao and H. Zhou, "Implementation of the measurement method and system for transient high-temperature based on colorimetric temperature measurement", Automation \& Instrumentation, vol. 9, pp. 5-8, 2010.

[16] J. Zhang and S. Yuan, "Application of RTD temperature measurement technology in compressor test", Aero-engine, vol. 4, pp. 48-60, 1994

[17] Z. Zhan, F. Ning, and J. Ding, "Study on some measuring technologies of temperature distortion on inlet flow of engine", Journal of Data Acquisition and Processing, vol. 15, no. 2, pp. 226-230, 2000

[18] D. Li, Research on the inlet flow field distortion temperature effects on aviation engine performance. University of Electronic Science and Technology of China: Chengdu, 2009.

[19] M. Zheng, Y. Wu and G. Zhao, "Transient temperature estimation for active-flow after-treatment control", Proceedings of ICES2005, vol. 1083, pp. 61-66, 2005

[20] P.C. Hung, G. Irwin and R. Kee, "Difference equation approach to two-thermocouple sensor characterization in constant velocity flow environments", Review of Scientific Instruments, vol. 76, no. 2:,pp. 024902-1-024902-10, 2005.

This is an open access article licensed under the terms of the Creative Commons Attribution Non-Commercial License (http://creativecommons.org/licenses/by-nc/4.0/) which permits unrestricted, non-commercial use, distribution and reproduction in any medium, provided the work is properly cited. 